Dansk Ultralyddiagnostisk Selskab

\title{
Virtual Reality - the future of ultrasound training, or just a gimmick?
}

Jonas D. Larsen ${ }^{1}$, Ole Graumann ${ }^{1,2}$, Rune Jensen ${ }^{1}$

\section{${ }^{1}$ Research and Innovation Unit of Radio- logy, Department of Clinical Research, University of Southern Denmark, Odense, Denmark \\ ${ }^{2}$ Department of Radiology, Odense Univer- sity Hospital, Odense, Denmark}

For clinical diagnostic and monitoring, point-of-care ultrasound (PoCUS) has become a useful tool [1]. PoCUS can support clinical decision making, but for ultrasoundnovices adequate knowledge, hands-on training and proper supervision are essential. With portable ultrasound scanners becoming increasingly available to clinicians, training is more crucial than ever before to prevent the clinical use outpacing evidencebased education and training. Structured ultrasound courses are already available in many locations. However, day-to-day training during clinical practice is still the main source for developing expertise in specialized ultrasound examinations [2, 3]. Major challenges with this approach are the random prevalence of specific pathologies and insufficient formal supervision/feedback due to limited resources.

Screen-based Virtual Reality (VR) simulation training ( $\triangleright$ Fig. $1 \mathrm{~A}$ ) has partially been used to overcome these educational challenges. Screen-based VR simulators often consist of a physical phantom simulating the patient and a computer, generating ultrasound images based on the movement of the ultrasound probe. These simulators provide a safe, controlled, and risk-free learning environment, where "patients" with different abnormal sonographic findings can be scanned repeatedly. Such setups, however, often require several physically large remedies and are resourceheavy with pricing up to $90000 €[4]$. Their accessibility is therefore severely limited and there is a need for more accessible VR-simulators.

Immersive Virtual Reality (IVR) based on head-mounted displays (HMD) is a new simulation modality that has shown great potential for medical education and acquisition of skills [5-9]. IVR uses software to create immersive 3-dimensional interactive environments which can be further enhanced by using a HMD with 360-degree stereoscopic field of view ( Fig. 1B, C). In 2013 Oculus Rift introduced a new generation of consumer-priced IVR-HMDs, and since then many other IVR-HMDs have become available, creating a nuanced and affordable market for research and education, with pricing from $300 €$. IVR-HMDs are portable, easily accessible, require few remedies to work ( 1 headset and 2 controllers) and are designed to be simple and safe to use. IVRHMD's can simulate any clinical setting and any clinical procedure in a small space of $2 \times 2$ meters, even at home, with under

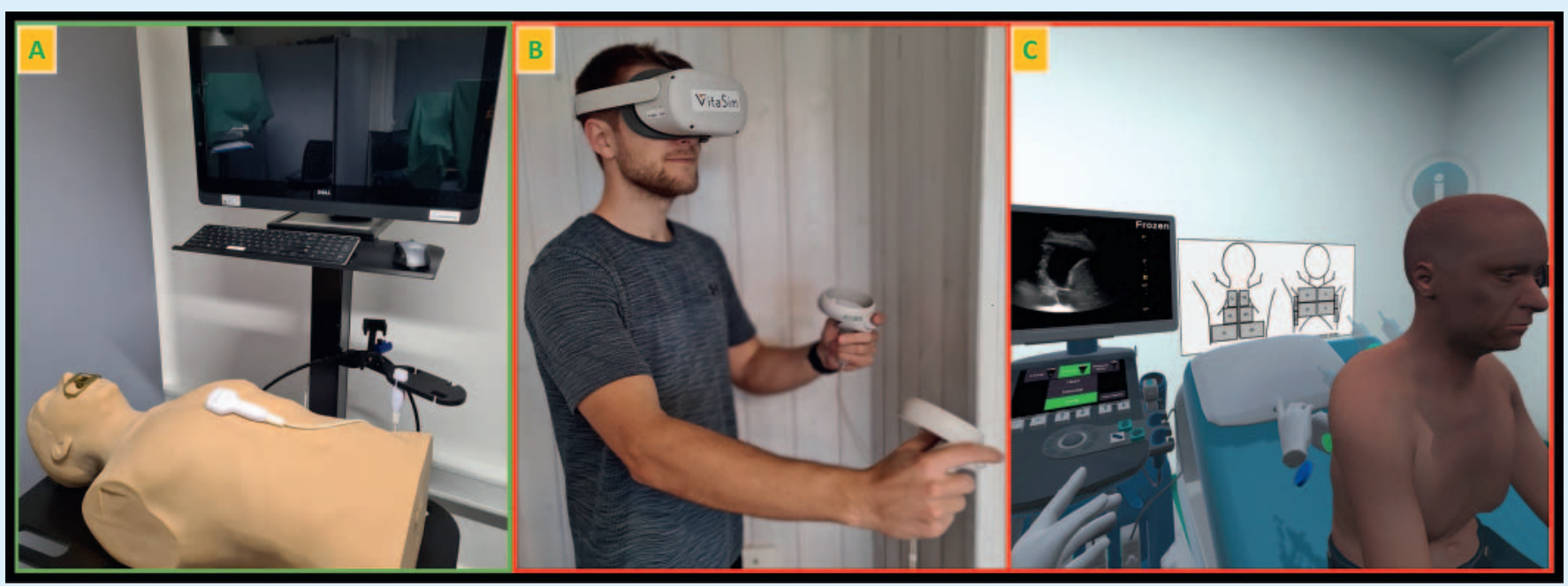

- Fig. 1 Illustrating the setups for different types of simulation training. A Screen based Virtual Reality simulation training. B Medical student equipped for IVR training ( 1 headset and 2 controllers). C Screenshot from inside the VR-HMD of how the student performs a sonographic examination in an IVR training room showing the patient (right), student holding the probe (middle), and the ultrasound machine displaying the patients sonographic images (left). 
5 minutes of setup, allowing the user to learn from experience in the virtual world. Like screen-based VR simulation training, IVR-HMD based training enables the user to gradually progress through different levels of difficulty. This can be performed in a realistic virtual learning environment with hundreds of different pathological cases (even rare ones) to experience and practice without bringing harm or nuisance to patients. Furthermore, software in IVR can provide automated, reliable, and structured feedback allowing the user to focus further training effort on identified pitfalls and personal challenges until an adequate level is obtained (e.g., correct interpretation of sonographic findings of a specific disease). Many of the advocating aspects for screen-based VR simulation training can therefore be recognized in IVR-HMD-based simulation training, but with greater accessibility and at a lower cost making it a beneficial supplement in ultrasound training.

A few studies have investigated the benefits of IVR-training in the acquisition of ultrasonographic competences, such as ultrasound-guided peripheral venous catheter placement and basic ultrasonographic skills $[10,11]$. The studies report positive learning outcomes but also agree that further research is needed to clarify the clinical implications of using IVR as a supplementary training tool for developing ultrasonographic competencies. It would be impractical for IVR to replace all aspects of sonographic training; hence, it should be emphasized to use the modality for specific and relevant learning goals such as scan- ning protocols, transducer handling, adjustment of gain, depth, focus, etc., and diagnostic capabilities. Other aspects of ultrasonographic training, such as acquiring theoretic baseline-knowledge about a certain scanning protocol or pathology, are probably not beneficial to learn using IVR and might not provide the same positive results compared to well-established sources for theoretical studies (e.g., e-learning).

The research in IVR-HMD-based simulation training will hopefully continue to push borders in a wide range of ultrasonographic settings. Aspects such as IVR-training in more advanced form of ultrasonographic skills (e.g., FLUS, FATE, FAST) as well as investigating the impact of such training on clinical and patient centred outcomes, should be explored in the future. Overall, IVR-HMD simulation training has shown great potential for improving training and education in ultrasound. However, implementation should be preceded by research focusing on targeting efficient aspects of ultrasonographic skills converted to IVRtraining, and the transfer of acquired skills into clinical practice.

\section{Reference}

[1] Kimura B]. Point-of-care cardiac ultrasound techniques in the physical examination: better at the bedside. Heart 2017; 103 (13): 987-994

[2] Organisation and practice of radiological ultrasound in Europe: a survey by the ESR Working Group on Ultrasound. Insights Imaging 2013; 4: 401-407
[3] Judmaier G. Training in ultrasound - can training standards be improved by exercises with a phantom. Ultraschall in Med 2003; 24: 231-233

[4] Østergaard ML, Konge L, Kahr N et al. Four Virtual-Reality Simulators for Diagnostic Abdominal Ultrasound Training in Radiology. Diagnostics (Basel) 2019; 9: 50

[5] Nickel F, Brzoska JA, Gondan M et al. Virtual reality training versus blended learning of laparoscopic cholecystectomy: a randomized controlled trial with laparoscopic novices. Medicine (Baltimore) 2015; 94: e764

[6] Huber T, Paschold M, Hansen C et al. New dimensions in surgical training: immersive virtual reality laparoscopic simulation exhilarates surgical staff. Surg Endosc 2017; 31: 4472-4477

[7] Hooper J, Tsiridis E, Feng JE et al. Virtual Reality Simulation Facilitates Resident Training in Total Hip Arthroplasty: A Randomized Controlled Trial. J Arthroplasty 2019; 34: 22782283

[8] Jensen L, Konradsen F. A review of the use of virtual reality head-mounted displays in education and training. Education and Information Technologies 2018; 23: 1515-1529

[9] Weiner E, Gordon J, Rudy S et al. Expanding Virtual Reality to Teach Ultrasound Skills to Nurse Practitioner Students. Stud Health Technol Inform 2019; 264: 893-897

[10] Andersen NL, Jensen RO, Posth S et al. Teaching ultrasound-guided peripheral venous catheter placement through immersive virtual reality: An explorative pilot study. Medicine (Baltimore) 2021; 100: e26394

[11] Rosenfeldt Nielsen M, Kristensen EQ, Jensen RO et al. Clinical Ultrasound Education for Medical Students: Virtual Reality Versus e-Learning, a Randomized Controlled Pilot Trial. Ultrasound Q 2021; 37: 292-296 